\title{
Phase-Space Semiclassical Analysis. Around Semiclassical Trace Formulae
}

\author{
Monique Combescure \\ Laboratoire de Physique Théorique, CNRS - UMR 8627 \\ Université de Paris XI, Bâtiment 210, F-91405 ORSAY Cedex, France \\ and \\ IPNL, Bâtiment Paul Dirac \\ 4 rue Enrico Fermi,Université Lyon-1 \\ F.69622 VILLEURBANNE Cedex, France \\ email monique.combescure@ipnl.in2p3.fr
}

\section{Résumé}

(1) What is semiclassical Analysis?

(2) What is a Trace Formula?

(3) Physical Motivations : "Quantum Chaos"

(4) Gutzwiller Trace Formula . (Rigorous Approach)

\section{COURS ÉCOLE D' ÉTÉ de CARGÈSE}

\section{"CHAOTIC DYMNAMICS; CLASSICAL AND QUANTUM TRANSPORT"}

Août 2003 


\section{CLASSICAL WORLD}

$Z=\mathrm{R}^{n} \times \mathrm{R}^{n}$ PHASE SPACE

$z:=(q, p)$ classical state of a particle

$q:$ position or coordinate

$\mathrm{p}:$ momentum

Hamiltonian $H=\frac{p^{2}}{2 m}+V(q)$

Real

Phase-Space Evolution :

$(q, p) \rightarrow\left(q_{t}, p_{t}\right)=\phi_{H}^{t}(q, p)$

$\phi_{H}^{t}$ preserves Phase-Space volumes

\section{Classical Observables}

$A: Z \mapsto \mathrm{R}$

Evolution of classical Observables

$A \circ \phi_{H}^{t}$

\section{Geometrical Tranformations}

- Phase-space translations

$\bullet, \quad J=\left(\begin{array}{cc}0 & \mathbb{1} \\ -\mathbb{1} & 0\end{array}\right)$ represents rotations of $\frac{\pi}{2}$

- General symplectic tranformations, ie

\section{QUANTUM WORLD}

$\mathcal{H}=L^{2}\left(\mathrm{R}^{n}\right)$ Hilbert space of quantum states

$\varphi \in \mathcal{H}$ "wavefunction"

$\hat{Q}$

$\hat{P}:=-i \hbar \nabla$ self-adjoint Operators

Quantum Hamiltonian $\hat{H}$ := $-\frac{\hbar^{2}}{2 m} \Delta+V(q)$

Self-Adjoint

Quantum evolution : $U_{H}(t):=$ $e^{-i t \hat{H} / \hbar}$

$\varphi \rightarrow \varphi_{t}:=U_{H}(t) \varphi$

Unitary Operator

Quantum Observables

$\hat{A}$ : Weyl Quantization of $A$

Evolution of Quantum Observables

$$
\hat{A}_{t}:=U_{H}^{*}(t) \hat{A} U_{H}(t)
$$

Unitary Transformations in $\mathcal{H}$

- $\hat{T}(z):=\exp (i \hat{Z} . J z / \hbar)$ WeylHeisenberg operator

- $\mathcal{F}$ Fourier Transformation

- General operators of metaplectic Group 


\section{CLASSICAL WORLD} (continued)

Preserve symplectic form $\sigma\left(z, z^{\prime}\right)=z . J z^{\prime}$

M : symplectic matrix $2 n \times 2 n$

Example $M=\left(\begin{array}{cc}e^{\gamma} \mathbb{1} & 0 \\ 0 & e^{-\gamma} \mathbb{1}\end{array}\right)$ dilation/squeezing transformation

\section{Multiplicative Group :} $M=M_{1} M_{2}$ symplectic

\section{CLASSICAL STATE}

Point $z \in Z$

\section{Probability Distribution} in PHASE SPACE :

$$
f: Z \mapsto \mathrm{R}^{+}
$$$$
f(q, p) \geq 0
$$

$$
\int_{Z} d z f(z)=1
$$

\section{QUANTUM WORLD (continued)}

$$
i \sigma(\hat{Z}, z) \text { generator of Weyl- }
$$

Heisenberg Group

$$
B: 2 n \times 2 n \text { real symmetric }
$$$$
M=e^{J B} \rightarrow \hat{R}(M)=e^{i \hat{Z} \cdot B \hat{Z} / 2 \hbar}
$$

$$
\left.\hat{D}(\gamma):=\exp \frac{i \gamma}{2 \hbar}(\hat{Q} \cdot \hat{P}+\hat{P} \cdot \hat{Q})\right)
$$

$\hat{R}\left(M_{1} M_{2}\right)=\hat{R}\left(M_{1}\right) \hat{R}\left(M_{2}\right)$ is Group

COHERENT STATE $|z\rangle$

\section{WIGNER, HUSIMI}

\section{distributions}

$$
\varphi \in \mathcal{H},\|\varphi\|=1
$$

Wigner Distribution

$$
\rightarrow W_{\varphi}(q, p)
$$

not $\geq 0$ in general

Husimi Distribution

$$
H_{\varphi}(z):=|\langle\varphi \mid z\rangle|^{2} \geq 0
$$

$\int_{Z} d z W_{\varphi}(z)=\int_{Z} d z H_{\varphi}(z)=1$ 


\section{What is Semiclassical Analysis?}

Semiclassical Analysis is the passage to the Limit $\hbar \rightarrow 0$ in the Quantum description in terms of the Evolution of states or Observables. It allows to better understand how the classical world description arises from a Quantum Reality.

However the limit $\hbar \rightarrow 0$ is a SINGULAR one, and in particular doesn't commute with the limit of large times $t \rightarrow \infty$, where classically long term UNPREDICTIBILITY ("chaos") manifests itself.

It will appear in what follows that Semiclassical Analysis (mathematical/physical) has to deal with estimates of Highly Oscillating Integrals with Small Parameter $\hbar$.

In what follows we shall present an illustration of Semiclassical Analysis which is simply an estimate of the Semiclassical Propagation of the so-called coherent states using the material presented in the comparative tableau above. The coherent states, usually denoted $\varphi_{z}$ in the mathematical notation, or $|z\rangle$ in the Dirac notation familiar to physicists are :

$$
|z\rangle:=\hat{T}(z)|0\rangle
$$

where :

$$
|0\rangle(x):=(\pi \hbar)^{-n / 4} \exp \left(-x^{2} / 2 \hbar\right)
$$

is the (normalized ) ground state of the Harmonic Oscillator

$$
\hat{H}_{0}=\frac{\hat{P}^{2}+\hat{Q}^{2}}{2}
$$

Let $\hat{H}$ be the Weyl quantization of a classical symbol, possibly depending on time $H(z, t)$ which satisfies the following assumption :

$$
\exists m, M, K>0: \quad\left(1+z^{2}\right)^{-M / 2}\left|\partial_{z}^{\gamma} H(z, t)\right| \leq K \quad \forall|\gamma| \geq m
$$

uniformly for $(z, t) \in[-T, T] \times Z$

such that the classical and quantum evolutions respectively (for the classical symbol and its Weyl quantization resp.) exist for $t \in[-T, T]$.

Then it is well known that the stability around a trajectory of the classical flow $z \rightarrow z_{t}$ is governed by the following symplectic matrix $F_{t}$ obeying the linear equation :

$$
\dot{F}=J M_{t} F
$$

where $M_{t}$ is the $2 n \times 2 n$ Hessian matrix of $\mathrm{H}$ at point $z_{t}$ of the classical trajectory :

$$
\left(M_{t}\right)_{j, k}:=\left(\frac{\partial^{2} H}{\partial z_{j} \partial z_{k}}\right)_{j, k}\left(z_{t}, t\right)
$$


is symmetric real, and the initial datum is

$$
F(0) \equiv \mathbb{1}
$$

To this symplectic matrix can be associated a unitary operator in $\mathcal{H}$ of the metaplectic group (see tableau above) $\hat{R}\left(F_{t}\right)$. We denote by $S_{t}$ the classical action along the classical flow $z \rightarrow z_{t}$ :

$$
S_{t}(z):=\int_{0}^{t} d s\left(\dot{q}_{s} \cdot p_{s}-H\left(z_{s}, s\right)\right)
$$

and we define :

$$
\begin{gathered}
\delta_{s}:=S_{t}(z)-\frac{q_{t} \cdot p_{t}-q \cdot p}{2} \\
\Phi(z, t):=e^{i \delta_{t} / \hbar} \hat{T}\left(z_{t}\right) \hat{R}\left(F_{t}\right)|0\rangle
\end{gathered}
$$

which is, up to the phase, a squeezed state located around the phase-space point $z_{t}$ with a dispersion governed by the matrix $F_{t}$. We can prove the following estimate :

Theorem 1.1 Let $H$ be an Hamiltonian satisfying the assumptions (1.2) and the existence of classical and quantum flows for $t \in[-T, T]$. Then we have, uniformly for $(t, z) \in[-T, T] \times Z$ :

$$
\left\|U_{H}(t, 0) \varphi_{z}-\Phi(z, t)\right\| \leq C \mu(z, t)^{P}|t| \sqrt{\hbar} \theta(z, t)^{3}
$$

$P$ being a constant only depending on $M$ and $m$, and

$$
\begin{gathered}
\mu(z, t):=\operatorname{Sup}_{0 \leq s \leq t}\left(1+\left|z_{s}\right|\right) \\
\theta(z, t):=\operatorname{Sup}_{0 \leq s \leq t}\left(t r F_{s}^{*} F_{s}\right)^{1 / 2}
\end{gathered}
$$

The estimate (1.9) contains the dependance in $t, \hbar, z$ of the semiclassical error term. One hopes that this error remains small when $\hbar \rightarrow 0$, provided that $\mathrm{z}$ belongs to some compact set of phase-space, and $|t|$ is not too large.

Typically

$$
\theta(z, t) \simeq e^{t \lambda}
$$

where $\lambda$ is some Lyapunov exponent that expresses the "classical instability" near the classical trajectory. The RHS of equ. (3.36) is therefore $O\left(\hbar^{\epsilon / 2}\right)$ provided

$$
|t|<\frac{1-\epsilon}{6 \lambda} \log \hbar^{-1}
$$

which is typically the Ehrenfest time, up to a factor $1 / 6$ that is probably inessential.

For more details and prior references see [6]. 


\section{What is a Trace Formula?}

Let $\mathcal{H}:=L^{2}\left(\mathrm{R}^{n}\right)$ be the Hilbert space of Quantum States, and $\hat{H}$ be a selfadjoint quantum Hamiltonian acting on it. It genarates via Schrödinger Equation an unitary quantum evolution operator

$$
U(t):=\exp (-i t \hat{H} / \hbar)
$$

We assume that the spectrum of $\hat{H}$ is pure point, namely :

$$
s p(\hat{H})=\left\{\lambda_{k}\right\}_{k \oplus \mathrm{N}}
$$

Then for $f$ sufficiently smooth and decreasing at infinity, $f(\hat{H})$ is a Trace Class Operator in $\mathcal{H}$. (it is enough for this that $\left\{f\left(\lambda_{k}\right)\right\}_{k \in N} \in l^{1}$ ), and we have :

$$
f(\hat{H}):=\sum_{k \in \mathrm{N}} f\left(\lambda_{k}\right)
$$

The Trace Formula is simply :

$$
\operatorname{tr} f(\hat{H})=\text { something else }
$$

\subsection{First Prototype : The Poisson summation Formula}

Under suitable definition of the Fourier Transform $f \rightarrow \tilde{f}$, we have :

$$
\sum_{n=-\infty}^{+\infty} f(n)=\sum_{k=-\infty}^{+\infty} \tilde{f}(2 k \pi)
$$

which holds true for any $f \in \mathcal{S}\left(\mathrm{R}^{n}\right)$ (that implies of course that also $\tilde{f} \in \mathcal{S}\left(\mathrm{R}^{n}\right)$ ), and which is therefore perfectly symmetric between $f, \tilde{f}$.

\section{Why do we mean that it is a Trace Formula?}

Consider the quantum operator in dimension $1: \hat{P}=-i \frac{d}{d x}$ acting on $L^{2}([0,2 \pi])$, with periodic boundary conditions $u(0)=u(2 \pi)$. It is an unbounded operator, whose spectrum if purely discrete :

$$
s p(\hat{P})=\mathrm{Z}
$$

Therefore the LHS of equ. (4.1) is nothing but $\operatorname{tr} f(\hat{P})$.

What does the RHS represents physically?

Imagine a classical Hamiltonian $H(q, p):=p$, where $q \in[0,2 \pi] .(\hat{P}$ is actually the Weyl quantization (for $\hbar=1$ ) of $\mathrm{H}$ in $L^{2}([0,2 \pi])$ with Periodic Boundary conditions). 
The associated Hamilton's equations are

$$
\dot{q}=1, \dot{p}=0
$$

anf thus : $q=t \quad(\bmod 2 \pi)$

The classical trajectories are thus all closed ie periodic in phase space, and are k-repetitions of the primitive orbit of period $2 \pi, \quad \forall k \in \mathrm{Z}$. This means that the periods of the classical flow are of the form $2 k \pi, \quad k \in \mathrm{Z}$.

The summation Poisson Formula thus expresses that the trace of a function of a quantum Hamiltonian of a very peculiar form is the sum on the periodic orbits of the corresponding classical flow of the Fourier Transform of that function taken at the periods of the classical flow.

\subsection{Second Prototype : The Harmonic Oscillator in dimen- sion 1}

$$
\hat{H}_{0}:=\frac{\hat{P}^{2}+\hat{Q}^{2}}{2}
$$

acting in $L^{2}(\mathrm{R})$ has spectrum $n+\frac{1}{2}$ (here again we let $\hbar=1$ ), where $n \in \mathrm{N}$

Take $f \in \mathcal{S}([0,+\infty])$. Then obviously

$$
\operatorname{tr} f\left(\hat{H}_{0}\right)=\sum_{n=0}^{\infty} f\left(n+\frac{1}{2}\right)
$$

Replace $\mathrm{f}$ by $\hat{T}(q, 0) f=f(.+q)$ in equ. (5.1). It becomes

$$
\sum_{n \in \mathbb{Z}} f(n+q)=\sum_{k \in \mathbb{Z}} e^{2 i \pi k q} \tilde{f}(2 k \pi)
$$

Thus taking $q=1 / 2 \quad e^{i k \pi}=(-1)^{k}$ which yields :

$$
\sum_{n=0}^{\infty} f\left(n+\frac{1}{2}\right)=\sum_{k \in \mathbb{Z}}(-1)^{k} \tilde{f}(2 k \pi)
$$

Since the classical trajectories of the classical Harmonic Oscillator are of the form

$$
q(t)=A \sin (t+\alpha)
$$

every orbit is therefore periodic, with a period which is a k-repetition of the primitive orbit of period $2 \pi$. The periods of the closed orbits of the classical flow are thus $\{2 k \pi\}_{k \in \mathbb{Z}}$ 
We see here a factor $(-1)^{k} \equiv e^{(2 k) i \pi / 2}$ which is here the first manifestation of a "Maslov Index" $2 k$.

Here again, equ. (2.18) expresses that the trace of the function of a Quantum Hamiltonian can be written as a sum over the periodic orbits of the corresponding classical flow of the Fourier Transform of that function, taken at the periods of the classical flow, up to some factor of the form $e^{\sigma_{k} i \pi / 2}$ where $\sigma_{k}$ is the Maslov index of the orbit.

The important fact to notice is that "someting else" in the RHS of (2.4) is a sum over the periodic orbits of the classical flow, and that the miracle obtained above in the two Prototypes has a Prolongation in the semiclassical limit, as we shall see in the last Section.

\section{Physical Motivations}

Consider as an illustration the case of billiards in $\mathrm{R}^{2}$ which are bounded domains $\Omega \in \mathrm{R}^{2}$ with a boundary denoted $\partial \Omega$ which is piecewise regular. Then two a priori not connected sets of problems can be addressed :

-on the Classical Side consider a point particle moving inside $\Omega$ with constant velocity, and specular reflections on the boundary $\partial \Omega$. We interest ourselves to the "spectrum" of lengths of periodic orbits inside the billiard. How are they distributed?

-on the other hand the equivalent of a Quantum Problem is the study of the Dirichlet Laplacian in $\Omega$. What is its spectrum and how is it distributed for large energies?

Is there a link between these two Problems? The answer is YES, semiclassically (that means asymptotically in the quantum spectrum). It somehow provides an answer of the famous V. Kac Question :

\section{CAN WE HEAR THE SHAPE OF A DRUM?}

or of a paraphrased problem raised by C.A. Pillet :

\section{CAN WE SEE THE SOUND OF A DRUM?}

The energy level density (defined in a distributional sense on suitable test functions) is the following :

$$
d(E):=\sum_{k \in \mathrm{N}} \delta\left(E-\lambda_{k}\right)
$$


Coming back to the traditional Schrödinger Hamiltonian, given E we can perform an average of $d(E)$ in a small neighborhood, which leads to the so-called "mean level density" $\bar{d}(E)$, and the first question to be addressed is that of finding a semiclassical approximation of it.

According to H. Weyl, we get :

$$
\int \bar{d}(E) \simeq \frac{\operatorname{vol}\left(\text { energy shell } \Sigma_{E}\right)}{h^{n}}
$$

where as usual :

$$
h:=2 \pi \hbar \rightarrow 0
$$

and where the energy shell is :

$$
\Sigma_{E}:=\{z: H(z)=E\}
$$

where $H$ is the classical symbol of $\hat{H}$.

The physical intuition for it is that the LHS of (3.20) "counts" the average number of states at energy $E$, and that one quantum state occupies a volume of phase-space of size $h^{n}$

The next section to be raised is the nature of the quantum fluctuations of $d(E)$ around $\bar{d}(E)$. These appear to be different according to whether the classical dynamics generated by $H$ is regular, (integrable), or chaotic.

-in the integrable case, trace formulae can be obtained and the semiclassical behavior of $d(E)-\bar{d}(E)$ can be expanded as a "sum" over the invariant tori of the classical flow, as obtained heuristically by Berry-Tabor ([2]), and rigorously by Colin de Verdière ([4]) in the framework of compact manifolds.

-in the chaotic case as a sum over the unstable periodic of the classical flow, as suggested by Balian-Bloch ([1]), and Gutzwiller ([8]).

Notice that in the two last works cited, the proof is heuristic, the sum over the periodic orbits is divergent, and the correction terms in $\hbar$ are omitted. Rigorous proofs have been established and are due to Chazarain [3], Duistermaat-Guillemin [7], Paul-Uribe [10], Meinrenken [9], and Combescure-Ralston-Robert [5]. All these proofs, as an assumption, take the Gutzwiller Hypothesis that the unstable Periodic Orbits are nondegenerate, or a weaker assumption of "clean flow" , and furthermore violate the beautiful classical/quantum duality of the exact Trace Formulae, in that the test functions $\varphi$ considered are assumed to have compact support in Fourier variable, which automatically truncates the sum over periodic orbits to those for which the period is in the support of $\tilde{\varphi}$. 


\section{Gutzwiller Trace Formula (Rigorous Proof)}

The proof we want to present here is the simplest one which makes use of semiclassical estimates of the evolution of coherent states, as presented in Section 1. (Theorem 1.1). The main mathematical trick is the following :

$$
\operatorname{tr} \hat{A}=h^{-n} \int_{Z} d z\langle z|\hat{A}| z\rangle
$$

for any trace class operator $\hat{A}$, and thus the following :

$$
\begin{gathered}
\operatorname{tr} \varphi\left(\frac{\hat{H}-E}{\hbar}\right)=\operatorname{tr}\left(\int d t e^{i t(E-\hat{H}) / \hbar} \tilde{\varphi}(t)\right) \\
=h^{-n} \int d t e^{i t E / \hbar} \tilde{\varphi}(t) \int_{Z} d z\left\langle z\left|e^{-i t \hat{H} / \hbar}\right| z\right\rangle
\end{gathered}
$$

We shall now make precise our assumptions on $H$ :

\section{Assumptions}

(H1) $H(\hbar, z) \asymp \sum_{j=0}^{\infty} \hbar^{j} H_{j}(z) \quad H_{j}: Z \mapsto \mathrm{R} \in \mathcal{C}^{\infty}(Z)$

(H2) $H_{0}$ bounded from below and

$$
0 \leq H_{0}(z)+\gamma_{0} \leq C\left(H_{0}\left(z^{\prime}\right)+\gamma_{0}\right)\left(1+\left|z-z^{\prime}\right|\right)^{M} \quad \forall z, z^{\prime} \in Z
$$

(H3) $\forall j \in \mathrm{N} \forall \gamma \in \mathrm{N}^{2 n} \quad\left|\partial_{z}^{\gamma} H_{j}(z)\right| \leq C\left(H_{0}(z)+\gamma_{0}\right)$ $z \in Z$

(H4) $\left|\partial_{z}^{\gamma}\left(H(\hbar, z)-\sum_{0}^{N} \hbar^{j} H_{j}(z)\right)\right| \leq C(N, \gamma) \hbar^{N+1} \quad \forall \hbar \in(0,1)$ uniformly for

(H5) Let $\left.I_{c l}:=\right] \lambda_{-}, \lambda_{+}$[ be an interval of classical energy, then $H_{0}^{-1}\left(I_{c l}\right)$ is bounded in $\mathrm{R}^{2 n}$ (the energy surfaces for $E \in I_{c l}$ are all compact)

(H6) $\mathrm{E}$ is a noncritical value for $H_{0}$ namely $H_{0}(z)=E \Rightarrow \nabla_{z} H_{0} \neq 0$

We can prove the following result:

Theorem 4.1 Let $H$ satisfy (H1)-(H6), and $\hat{H}$ its Weyl quantization. Consider the classical flow $\phi_{H_{0}}^{t}$ of $H_{0}$ on $\Sigma_{E}:\left\{H_{0}(z)=E\right\}$, and denote by $\gamma$ the periodic orbits of this flow. We assume in addition (Gutzwiller Hypothesis) that the Poincaré map 
$P_{\gamma}$ dosn't have 1 as eigenvalue, namely that the orbits $\gamma$ are nondegenerate. Then for any $\varphi \in \mathcal{S}: \tilde{\varphi} \in \mathcal{C}_{0}^{\infty}$, we have, asymptotically as $\hbar \rightarrow 0$ :

$$
\begin{gathered}
\operatorname{tr} \varphi\left(\frac{\hat{H}-E}{\hbar}\right) \asymp h^{-n}\left(\tilde{\varphi}(0)\left|\Sigma_{E}\right| \hbar+\sum_{j \geq 2} \hbar^{j} c_{0, j}(\tilde{\varphi})\right) \\
+\sum_{\gamma} \frac{e^{i S_{\gamma} / \hbar}+i \sigma_{\gamma} \pi / 2}{\left|\operatorname{det}\left(\mathbb{1}-P_{\gamma}\right)^{1 / 2}\right|}\left(\tilde{\varphi}\left(T_{\gamma}\right) \frac{T_{\gamma}^{*}}{2 \pi} e^{-i \int_{\gamma} H_{1}}+\sum_{j \geq 1} \hbar^{j} c_{\gamma, j}(\tilde{\varphi})\right)
\end{gathered}
$$

where:

$\gamma^{*}$ is the primitive orbit corresponding to $\gamma$

$T_{\gamma}$ (resp. $\left.T_{\gamma}^{*}\right)$ is the period of $\gamma\left(\right.$ resp. $\left.\gamma^{*}\right)$

$\sigma_{\gamma}$ is the Maslov index of $\gamma$

$S_{\gamma}$ is the classical action along $\gamma$

$P_{\gamma}$ is the Poincaré map of $\gamma$

$c_{0, j}$ are distributions supported in $\{0\}$

$c_{\gamma, j}$ are distributions supported in $\left\{T_{\gamma}\right\}$

Note that the duality Quantum/Classical is violated by the condition that $\tilde{\varphi}$ is of compact support.

The First line of (5.7) corresponds to the "regular part" in $\hbar$, and we recognize the dominant Weyl term (with corrections in $\hbar$ that Gutzwiller had "omitted", or neglected...)

The Second line corresponds to the "oscillating part" in $\hbar$, which is, as expected, a sum over the periodic orbits of the classical flow (here truncated since $\tilde{\varphi}\left(T_{\gamma}\right)=0$ for $\gamma$ large).

\section{Références}

[1] Balian R., and Bloch C.; Distribution of eigenfrequencies for the wave equation in a finite domain, Ann. Phys. 69, vol.1, 76-160, (1972)

[2] Berry M. and Tabor, M. : Closed orbits and the regular bound spectrum, Proc. Roy .Soc. Lond, A349, 101-23, (1976)

[3] Chazarain J. : Formule de Poisson pour les variétés Riemanniennes. Inv. Math. 24,65-82, (1974)

[4] Colin de Verdière Y. : Spectre du Laplacien et longueurs des géodésiques périodiques I, Compos. Math. 27, 83-106, (1973) 
[5] Combescure M., Ralston J., and Robert D. : A Proof of the Gutzwiller Semiclassical Trace Formula using Coherent States decomposition, Commun. Math. Phys. 202, 463-480, (1999)

[6] Combescure M., and Robert D. : Semiclassical spreading of quantum wavepackets and applications near unstable fixed points oif the classical flow, Asymptotic Anal. 14, 377-404, (1997)

[7] Duistermaat J. J., and Guillemin V. : The spectrum of positive elliptic operators and periodic bicharacteristics, Invent. Math. 29, 39-79, (1975)

[8] Gutzwiller M., Periodic orbits and the classical quantization condition, J. Math. Phys. 12, 343-358 (1971)

[9] Meinrenken E. : Semiclassical principal symbols and the Gutzwiller's trace formula, Reports on Math. Phys. 31, 279-295, (1992)

[10] Paul T. and Uribe A. : The semiclassical trace formula and propagation of wavepackets, J. Funct. Anal. 132, 192-249 (1996) 